ISSN 0103-5150

Fisioter. Mov., Curitiba, v. 25, n. 2, p. 369-377, abr./jun. 2012 Licenciado sob uma Licença Creative Commons

\title{
Avaliação da prevalência de osteoporose e fraturas vertebrais em pacientes portadores de doença pulmonar obstrutiva crônica
}

\author{
Evaluation of the prevalence of osteoporosis and vertebral \\ fractures in patients with chronic obstructive pulmonary disease
}

\author{
Gualberto Ruas $^{[\mathrm{a}]}$, Marcelo Cunha Fatureto ${ }^{[\mathrm{b}]}$, José Henrique Santana ${ }^{[\mathrm{c}]}$, \\ Valéria Amorim Pires di Lorenzo ${ }^{[\mathrm{d}]}$, Mauricio Jamami ${ }^{[\mathrm{d}]}$ \\ [a] Fisioterapeuta, Doutorando do Programa de Pós-Graduação em Fisioterapia da Universidade Federal de São Carlos \\ (UFSCar), São Carlos, SP - Brasil, e-mail: gualbertoruas@yahoo.com.br \\ [b] Médico, professor adjunto responsável pela disciplina de Cirurgia Torácica da Universidade Federal do Triângulo Mineiro \\ (UFTM), Uberaba, MG - Brasil. \\ [c] Médico, professor Doutor adjunto, chefe da disciplina de Pneumologia do Departamento de Clínica Médica da \\ Universidade Federal do Triângulo Mineiro (UFTM), Uberaba, MG - Brasil. \\ [d] Fisioterapeutas, professores Doutores adjuntos da Graduação e do Programa de Pós-Graduação em Fisioterapia \\ (Departamento de Fisioterapia) da Universidade Federal de São Carlos (UFSCar), São Carlos, SP - Brasil.
}

\section{Resumo}

Introdução: A osteoporose é uma doença progressiva que contribui para o surgimento de fraturas vertebrais. Objetivos: Avaliar a prevalência da osteoporose, fraturas vertebrais e intensidade da dor nas regiões da coluna vertebral em pacientes com DPOC moderada e grave, além de verificar se há relação entre tais variáveis e a gravidade da obstrução pulmonar. Materiais e métodos: Foram avaliados 34 indivíduos portadores de DPOC (GDPOC) e 33 indivíduos saudáveis (GC), por meio da densitometria óssea e radiografias da coluna torácica e lombar. Resultados: Não houve diferenças significativas intergrupos nos dados antropométricos. O GDPOC apresentou valores menores nas variáveis espirométricas, na densidade mineral óssea (DMO) e no escore-T, quando comparado com o GC ( $\mathrm{p} \leq 0,05$; Teste $t$ Student); além disso, não se observou correlação entre $\mathrm{VEF}_{1}$ e DMO e escore-T (Correlação de Pearson, p > 0,05). Verificou-se uma alta prevalência de osteoporose, sendo que 20 indivíduos (59\%) com obstrução moderada apresentaram na coluna lombar, l (3\%) no colo do fêmur e l (3\%) no trocânter; 4 (12\%) com obstrução pulmonar grave na coluna lombar, l (3\%) no colo do fêmur e l (3\%) no trocânter. Vinte indivíduos (59\%) do GDPOC com osteoporose 
apresentaram fraturas de graus I e II, 4 (12\%) grau III ( $\mathrm{k}=$ 0,90; IC: 95\%) e 3 indivíduos do GDPOC relataram presença de dor com intensidade "leve" na região toracolombar, enquanto 21 apresentaram fraturas vertebrais osteoporóticas assintomáticas. Conclusão: Concluímos que os indivíduos com DPOC de moderada a grave apresentaram, além do comprometimento pulmonar, diminuição da DMO e fraturas vertebrais osteoporóticas, o que indica a necessidade de se adotar medidas de prevenção da osteoporose nesses pacientes.

Palavras-chave: Densidade mineral óssea. Prevenção. Fatores de risco. Dor. Fisioterapia.

\begin{abstract}
Introduction: Osteoporosis is a progressive disease that contributes to the emergence of vertebral fractures. objectives: To evaluate the prevalence of osteoporosis, vertebral fractures and pain intensity in the regions of the spine in patients with moderate or severe COPD and to check whether there is a connection between these variables and gravity of pulmonary obstruction. Materials and methods: We evaluated 34 subjects with COPD (COPDG) and 33 healthy subjects (CG), using bone densitometry and radiographs of the thoracic and lumbar spine. Results: There were no significant differences between groups in demographics. The COPDG values were lower in spirometric variables in bone mineral density (BMD) and T-score compared to the $C G(p<0.05$, Student's t test) and, moreover, no correlation was observed between BMD and FEV $V_{1}$ and T-score (Pearson correlation, $p>0.05)$. There was a high prevalence of osteoporosis, and 20 individuals (59\%) presented with moderate obstruction in the lumbar spine, l (3\%) in the femoral neck and l (3\%) trochanter; 4 (12\%) with severe pulmonary obstruction in the lumbar spine, $l(3 \%)$ in the femoral neck and $l(3 \%)$ in the trochanter. Twenty subjects (59\%) of COPDG osteoporotic fractures had grade I and II, 4 (12\%) grade III ( $k=0.90$, CI: 95\%) and three individuals COPDG reported presence of "light" pain intensity in the thoracolumbar region, while 21 had asymptomatic osteoporotic vertebral fractures. Conclusion: We conclude that individuals with moderate to severe COPD showed beyond the pulmonary obstruction, decreased BMD and osteoporotic vertebral fractures, which indicates the need to adopt measures to prevent osteoporosis in these patients.
\end{abstract}

Keywords: Bone mineral density. Prevention. Risk factors. Pain. Physiotherapy.

\section{Introdução}

A osteoporose é uma doença óssea progressiva primária ou secundária, caracterizada pela diminuição da massa óssea e alterações das microarquiteturas dos tecidos ósseos, com aumento da fragilidade mecânica óssea e predisposição às fraturas (1). É considerada um problema importante de saúde pública, pois grande parte da população mundial apresenta a doença, calculando-se que cerca de 10 milhões de pessoas entre homens e mulheres estejam afetadas (2).

Apesar de ser considerada uma doença de mulheres, vários estudos têm mostrado uma alta prevalência em homens (3-7) por causas secundárias que interferem direta ou indiretamente no metabolismo ósseo, sendo as mais frequentes (30\% a 60\%) (8), o uso de glicocorticoide, tabagismo, doença pulmonar obstrutiva crônica (DPOC), hipogonadismo e o alcoolismo (9).

Estudos realizados em portadores de DPOC evidenciam que, com a progressão da doença e debi- lidade, os pacientes apresentam uma redução significativa e importante da densidade mineral óssea (DMO) em virtude de vários outros fatores de risco associados, como a desnutrição, a redução da massa muscular e o sedentarismo, podendo contribuir para outras complicações tais como as fraturas osteoporóticas (10-12).

A principal complicação nesses pacientes é a fratura vertebral, que contribui para o surgimento de dor, redução da capacidade de exercício e piora da função pulmonar, sendo que cada vértebra torácica fraturada leva a uma diminuição de aproximadamente $9 \%$ na capacidade vital forçada (13).

Segundo Soriano et al. (14), os pacientes com diagnóstico recente de DPOC apresentaram três vezes mais risco de osteoporose e 1,6 vezes mais risco de fraturas quando comparados com o grupo controle.

Atualmente, as fraturas vertebrais osteoporóticas são as menos estudadas, e os dados sobre sua ocorrência nesses pacientes são escassos e limitados, já 
que na maioria das vezes os pacientes não apresentam sintomas, oferecendo um risco substancial não só para novas fraturas, mas consequências clínicas e piora do estado geral de saúde. Diante do exposto, há a necessidade de estudos que identifiquem a presença da osteoporose e fraturas vertebrais em pacientes com DPOC, utilizando métodos eficazes e validados.

Dessa forma, o presente estudo teve como objetivos avaliar a prevalência da osteoporose, fraturas vertebrais e intensidade da dor nas regiões da coluna vertebral em pacientes com DPOC moderada e grave, além de verificar se há relação entre tais variáveis e a gravidade da obstrução pulmonar. Assim, seria possível contribuir para que os profissionais adotassem medidas de prevenção da osteoporose nesses pacientes e fizessem o diagnóstico precoce da doença. Além disso, auxiliaria o profissional fisioterapeuta a estabelecer um programa de intervenção adequado.

\section{Materiais e métodos}

\section{Casuística e aspectos éticos}

Foi realizado um estudo transversal e duplo cego, em indivíduos encaminhados ao serviço de Fisioterapia Respiratória desta instituição. Em atendimento à Resolução n. 196/96 do Conselho Nacional de Saúde, o estudo foi aprovado pelo Comitê de Ética e Pesquisa (parecer n. 151/2009); e todos assinaram um termo formal de consentimento livre e esclarecido.

Inicialmente, foram avaliados 70 homens, sendo 35 portadores de DPOC com obstrução moderada a grave compondo o grupo DPOC (GDPOC) e 35 saudáveis sem comprometimento respiratório compondo o grupo controle (GC).

No GDPOC apenas um indivíduo foi excluído por faltar mais de três vezes nas datas agendadas para avaliação e por apresentar diagnóstico médico de insuficiência renal crônica e diabetes mellitus tipo l. Concluíram as avaliações 34 indivíduos, sendo 28 com obstrução moderada e 6 com grave. No GC apenas 2 indivíduos foram excluídos por não completarem a avaliação; sendo assim, 33 concluíram todas as avaliações.

\section{Critérios de inclusão e exclusão}

No GDPOC, como critérios de inclusão, foram considerados aptos a participar indivíduos do sexo mas- culino com diagnóstico clínico de DPOC apresentando $\mathrm{VEF}_{1} / \mathrm{CVF}<70 \%$ e $30 \% \leq \mathrm{VEF}_{1}<80 \%$ do previsto constatado pela espirometria, ex-tabagistas, com uso de corticoides sistêmicos e/ou orais por mais de três meses em dose diária superior a $5 \mathrm{mg}$ de prednisona ou equivalente - expostos à dose cumulativa de prednisona ou equivalente $\geq 1.000 \mathrm{mg}$ ao longo da vida $(15,16)$, sem histórias de infecções ou exacerbações dos sintomas respiratórios nos últimos três meses, sem fraturas traumáticas, clinicamente estáveis, sem comprometimentos cardiovasculares, neurológicos e/ou alterações cognitivas que comprometessem a avaliação. Para o GC os critérios foram: indivíduos do sexo masculino, função pulmonar dentro dos padrões de normalidade constatada pela espirometria, sem infecções respiratórias ou doenças cardíacas e reumáticas, não tabagistas ou ex-tabagistas e clinicamente estáveis.

Foram excluídos os indivíduos que apresentaram história de fraturas traumáticas e atraumáticas nas regiões da coluna.

\section{Procedimento experimental}

As cinco avaliações ocorreram em dias distintos e não consecutivos, sendo compostas por:

\section{Exames bioquímicos (séricos)}

Todos os indivíduos foram submetidos à coleta de amostras de sangue e urina solicitadas por um médico hematologista e um pneumologista, e orientados a permanecerem em jejum por 12 horas para a dosagem dos níveis de vitamina $\mathrm{D}$, testosterona, cálcio, fósforo, fosfatase alcalina, albumina e calciúrina de 24 horas para dosagem do cálcio e das proteínas. Todos os procedimentos seguiram as rotinas do laboratório responsável.

\section{Espirometria}

Todos os indivíduos do GDPOC e GC realizaram um teste espirométrico seguindo as Diretrizes da Sociedade Brasileira de Pneumologia e Tisiologia (17), sendo obtidas as medidas de capacidade vital lenta (CVL), capacidade vital forçada (CVF), volume expiratório forçado no primeiro segundo $\left(\mathrm{VEF}_{1}\right)$, relação 
volume expiratório forçado no primeiro segundo e capacidade vital forçada $\left(\mathrm{VEF}_{1} / \mathrm{CVF}\right)$, pico de fluxo expiratório (PFE), fluxo expiratório forçado $25-75 \%$ (FEF25-75\%) e ventilação voluntária máxima (VVM). Os resultados do teste de função pulmonar foram comparados com os valores previstos de Knudson (1983).

\section{Densitometria óssea}

A DMO foi determinada pelo sistema Dual Energy X-ray Absorptiometry, em equipamento Lunar ${ }^{\circledR}$ Excellent in Imaging versão 4.7e, Madison, Wisconsin, EUA, devidamente calibrado e submetido ao controle de qualidade diariamente, conforme especificações do fabricante. Todas as avaliações foram realizadas por um técnico habilitado em radiologia médica. Obtiveram-se valores da densidade mineral óssea em vários sítios esqueléticos, tais como: coluna lombar de $\mathrm{L}_{2}$ a $\mathrm{L}_{4}$ ântero-posterior, fêmur proximal incluindo o colo do fêmur e o trocânter, todos expressos $\mathrm{em} \mathrm{g} / \mathrm{cm}^{2}$; e valor de escore-T que reflete o número de desvios padrão (DP) em que o valor da DMO do paciente difere do valor de adultos jovens. Os valores de DMO para indivíduos normais do sexo masculino foram obtidos do banco de dados padronizado do NHANES III (18). O coeficiente de variação (precisão-CV\%) in vitro do equipamento, obtido em um bloco de alumínio (phanton), é de $0,81 \%$. 0 CV\% in vivo é de 0,9-1,1\% para a coluna lombar (mínima variação significativa de $2,9 \%$ ) e de $0,9-1,3 \%$ para o colo do fêmur (mínima variação significativa de 3,5\%).

\section{Morfometria vertebral}

As radiografias foram realizadas na coluna torácica e lombar, com os filmes centrados, e direção dos raios-X, em $\mathrm{T}_{7}$ e $\mathrm{L}_{2}$, respectivamente. Ambas foram obtidas com o indivíduo na posição lateral esquerda, adicionando-se a técnica de inspiração para aquelas relacionadas à coluna torácica (19-20).

A morfometria vertebral foi obtida utilizando-se um cursor translúcido para a marcação de cada corpo vertebral em seis pontos: dois anteriores, dois médios e dois posteriores, segundo os critérios adotados mundialmente para a definição de fraturas (21-22), pela medida da altura anterior, média e posterior dos corpos vertebrais de $\mathrm{T}_{4}$ a $\mathrm{L}_{5}$. E a partir dessa definição, foram classificados de acordo com os graus descritos:

Grau I: "Leve" fratura de compressão com diminuição da altura anterior maior que $20 \%$ e menor que $25 \%$.

Grau II: "Moderada" fratura de compressão com diminuição da altura anterior maior que $25 \%$ e menor que $40 \%$, ou deformidade entre $20 \%$ e $25 \%$, atingindo a altura posterior ou média.

Grau III: "Severa" fratura de compressão com intensa deformidade e perda de volume ou área projetada maior que $40 \%$ relativa à vértebra adjacente não fraturada.

A análise radiográfica foi realizada de forma cega por dois examinadores - sendo um médico radiologista e um fisioterapeuta - para observar o nível de concordância entre eles. Utilizou-se um sistema de alta resolução digital (PACS - Sistema de Comunicação e Arquivamento de Imagens) da marca Agfa ${ }^{\circledR}$ Medical Systems, versão: 3.3.16, Bélgica.

\section{Avaliação do nível de dor}

Todos os indivíduos foram avaliados quanto à presença e à intensidade da dor nas regiões da coluna cervical, torácica e lombar. Foi utilizada a Escala de Borg Category-Ratio (EB CR-10) (23), que possui uma pontuação que vai de 0 a 10 , na qual 0 (zero) representa nenhum sintoma e 10 representa sintoma máximo. Todos foram orientados para escolher uma única pontuação que refletisse a intensidade dessa dor.

\section{Análise estatística}

Para a análise dos resultados, utilizou-se o programa estatístico InStat versão 3.05 (GraphPad Software Inc., San Diego, CA, EUA). As variáveis estudadas são apresentadas em seus valores de médias \pm desvios padrão.

Considerando a normalidade das variáveis, por meio do teste de Kolmogorov e Smirnov, foi utilizado o teste $t$-Student não pareado para a comparação entre os grupos e pareado para a comparação intragrupo.

Para o estudo das relações entre as variáveis foi utilizado o coeficiente de correlação de Pearson. 0 nível de significância considerado foi de $\mathrm{p} \leq 0,05$. 
0 cálculo do power da amostra foi realizado pelo programa GraphPad StatMate 2.0 para Windows. A amostra correspondeu a um power de $80 \%$ para as variáveis avaliadas. Além disso, foi realizada a análise estatística de Kappa de Cohen (23) para avaliar o nível de concordância entre os avaliadores na morfometria vertebral, considerando $\mathrm{k}=0$ (pobre); 0 a 0,20 (ligeira); 0,21 a 0,40 (considerável); 0,41 a 0,60 (moderada); 0,61-0,80 (substancial) e 0,81 a 1,0 (concordância excelente) (24).

\section{Resultados}

Observou-se no presente estudo que os exames bioquímicos (séricos) realizados por todos os indivíduos se encontravam dentro dos limites de normalidade e não houve diferenças significativas entre os grupos. As características antropométricas e os valores espirométricos dos indivíduos do GC e do GDPOC estão expostos na Tabela 1. Na análise intergrupos, verificou-se que não houve diferenças significativas das características antropométricas como idade, peso, altura e índice de massa corpórea (IMC) entre os grupos. No entanto, o GDPOC apresentou valores de $\mathrm{CVF}, \mathrm{VEF}_{1}, \mathrm{VEF}_{1} / \mathrm{CVF}$ e VVM significativamente menores que o GC (Teste $t$-Student; $\mathrm{p} \leq 0,05) \cdot 0 \mathrm{VEF}_{1}$ não apresentou correlação com a DMO nem com o escore T (Correlação de Pearson, $\mathrm{p}>0,05)$.

\section{DMO do GDPOC e do GC}

O GDPOC apresentou valores significativamente menores da DMO e do escore T nas regiões da coluna lombar, colo do fêmur e trocânter, quando comparado com o GC (Tabela 2).

Prevalência de osteoporose de acordo com o grau de obstrução no GDPOC

Observamos neste estudo que, dos 34 indivíduos com DPOC avaliados, 28 (83\%) possuem osteoporose (Tabela 3).

Tabela 1 - Características antropométricas e valores espirométricos dos indivíduos do GC e do GDPOC

\begin{tabular}{lcc}
\hline & $\begin{array}{c}\text { GC } \\
\mathbf{n}=\mathbf{3 3}(\mathbf{H})\end{array}$ & $\begin{array}{c}\text { GDPOC } \\
\mathbf{n}=\mathbf{3 4}(\mathbf{H})\end{array}$ \\
\hline Idade (anos) & $68 \pm 3$ & $69 \pm 8$ \\
Peso (kg) & $71 \pm 6$ & $65 \pm 7$ \\
Altura (cm) & $174 \pm 4$ & $170 \pm 6$ \\
IMC (kg/m²) & $23 \pm 2$ & $22 \pm 2$ \\
CVF (\%prev) & $92 \pm 15$ & $81 \pm 15^{*}$ \\
VEF $_{1}(\%$ prev) & $85 \pm 13$ & $61 \pm 14^{*}$ \\
VEF $/$ CVF (\%) & $92 \pm 5$ & $57 \pm 6^{*}$ \\
VVM (\%prev) & $85 \pm 13$ & $58 \pm 14^{*}$ \\
\hline
\end{tabular}

Fonte: Dados da pesquisa.

Legenda: Os dados estão expressos em média \pm desvio padrão; $\mathrm{GC}=$ grupo controle; GDPOC = grupo DPOC; $\mathrm{H}=$ gênero masculino; $\mathrm{kg}=$ quilograma; $\mathrm{cm}=$ centímetro; $\mathrm{kg} /$ $\mathrm{m}^{2}$ = quilograma por metro quadrado; $\mathrm{CVF}=$ capacidade vital forçada; $\mathrm{VEF}_{1}=$ volume expiratório forçado no primeiro segundo; $\mathrm{VEF}_{1} / \mathrm{CVF}=$ Relação volume expiratório forçado no primeiro segundo e capacidade vital forçada; VVM = ventilação voluntária máxima; \%prev = porcentagem do previsto; ${ }^{*}=p \leq 0,05$; Teste $t$-Student.

Tabela 2 - Médias e desvios padrão da DMO e escore T dos indivíduos do GC e do GDPOC das regiões avaliadas

\begin{tabular}{|c|c|c|c|c|}
\hline \multirow[b]{2}{*}{ Regiões } & \multicolumn{2}{|c|}{ GC } & \multicolumn{2}{|c|}{ GDPOC } \\
\hline & DMO $\left(\mathrm{g} / \mathrm{cm}^{2}\right)$ & escore T & DMO $\left(\mathrm{g} / \mathrm{cm}^{2}\right)$ & escore T \\
\hline Coluna lombar & $0,99 \pm 0,01$ & $-0,5 \pm 0,08$ & $0,68 \pm 0,1^{* \star *}$ & $-4,2 \pm 1^{\star \star \star}$ \\
\hline Colo do fêmur & $0,93 \pm 0,04$ & $-0,7 \pm 0,04$ & $0,74 \pm 0,1^{* * *}$ & $-2,75 \pm 0,1^{\star \star \star *}$ \\
\hline Trocânter & $0,94 \pm 0,02$ & $-0,9 \pm 0,02$ & $0,76 \pm 0,1^{* * *}$ & $-2,47 \pm 1^{\star \star *}$ \\
\hline
\end{tabular}

Fonte: Dados da pesquisa.

Legenda: $\mathrm{GC}=$ grupo controle; GDPOC = grupo DPOC; DMO = densidade mineral óssea; $\mathrm{g} / \mathrm{cm}^{2}=$ grama por centímetro ao quadrado; $* * *=$ significativo, $p \leq 0,01$; Teste $t$-Student. 
Presença de fraturas vertebrais no GDPOC de acordo com o grau de obstrução e no GC

Vinte indivíduos (59\%) do GDPOC com obstrução pulmonar moderada e quatro indivíduos (12\%) com grau de obstrução pulmonar grave portadores de osteoporose na coluna lombar apresentaram fraturas vertebrais osteoporóticas (Tabela 4).

No GC observamos que três indivíduos apresentaram fraturas vertebrais e artrose nos segmentos $\mathrm{T}_{5}, \mathrm{~T}_{6}, \mathrm{~L}_{1}$ e nenhum indivíduo apresentou fraturas vertebrais osteoporóticas ( $\mathrm{k}=0,89$; IC: $95 \% \operatorname{com~} \mathrm{k}=$ $0,72-1,0)$.

Intensidade da dor do GDPOC e GC

Três indivíduos do GDPOC com obstrução grave que apresentaram osteoporose e fraturas vertebrais osteoporóticas grau III relataram presença de dor com intensidade "leve" na região toracolombar, 21 indivíduos apresentaram fraturas vertebrais osteoporóticas assintomáticas, e três indivíduos do GC apresentaram fraturas vertebrais não osteoporóticas e artrose, relatando, também, dor de intensidade moderada na região lombar.

\section{Discussão}

Neste estudo verificou-se que a DMO e o escore $\mathrm{T}$ das regiões da coluna lombar, colo do fêmur e trocânter apresentaram-se significativamente menores no GDPOC quando comparados com o GC.

No que se refere à presença de osteoporose nos indivíduos com DPOC de obstrução moderada, no presente estudo foi de $83 \%$ (28 indivíduos), sendo $59 \%$ na coluna lombar; $3 \%$ no colo do fêmur e 3\% no trocânter. Quatro (12\%) indivíduos com DPOC com obstrução grave apresentaram osteoporose na coluna lombar, um (3\%) no colo do fêmur e um (3\%) no trocânter. Nesse sentido, Forli et al. (25), analisando a DMO numa população de candidatos a transplante pulmonar com doenças pulmonares de etiologias variadas e com uso contínuo de corticoide sistêmico, observaram que $90 \%$ dos indivíduos apresentavam uma queda significativa da DMO.

Estudos mostram que doses acumulativas maiores que $1.000 \mathrm{mg}$ de prednisona ou equivalente em indivíduos com DPOC $(15,26)$ são capazes de promover
Tabela 3 - Médias e desvios padrão da DMO e escore T dos indivíduos do GC e do GDPOC das regiões avaliadas

\begin{tabular}{lcc}
\hline & $\begin{array}{c}\text { Obstrução moderada } \\
(\mathbf{n}=\mathbf{2})\end{array}$ & $\begin{array}{c}\text { Obstrução grave } \\
(\mathbf{n}=\mathbf{6})\end{array}$ \\
\hline Coluna lombar & $20(59 \%)$ & $04(12 \%)$ \\
Colo do fêmur & $01(3 \%)$ & $01(3 \%)$ \\
Trocânter & $01(3 \%)$ & $01(3 \%)$ \\
\hline
\end{tabular}

Fonte: Dados da pesquisa.

Legenda: GDPOC = grupo DPOC; $\mathrm{n}=$ número de indivíduos; $\%=$ porcentagem.

Tabela 4 - Presença de fraturas vertebrais no GDPOC de acordo com a gravidade da obstrução

\begin{tabular}{lll}
\hline & \multicolumn{2}{c}{ Fraturas vertebrais (segmentos) e graus } \\
\cline { 2 - 3 } $\begin{array}{l}\text { Região com } \\
\text { osteoporose }\end{array}$ & $\begin{array}{c}\text { Obstrução moderada } \\
(\mathbf{n}=\mathbf{2 0 - 5 9 \% )}\end{array}$ & $\begin{array}{c}\text { Obstrução grave } \\
(\mathbf{n}=\mathbf{4}-\mathbf{1 2} \%)\end{array}$ \\
\hline Coluna lombar & $\begin{array}{l}\mathrm{T} 5, \mathrm{~T} 7, \mathrm{~T} 9, \mathrm{~T} 11, \mathrm{~T} 12 \text { e L1 } \\
(\mathrm{Gl} \text { e II) }\end{array}$ & $\begin{array}{l}\mathrm{T} 12, \mathrm{~L} 1, \mathrm{~L} 2, \mathrm{~L} 3 \text { e } \mathrm{L} 4 \\
(\mathrm{GIII})\end{array}$ \\
& $(\mathrm{k}=0,90 ;$ & $(\mathrm{k}=0,90 ;$ \\
& $\mathrm{IC}: 95 \%$ com & $\mathrm{IC}: 95 \%$ com \\
& $\mathrm{k}=0,72-1,0)$ & $\mathrm{k}=0,72-1,0)$ \\
\hline
\end{tabular}

Fonte: Dados da pesquisa.

Legenda: GDPOC = grupo DPOC; $\mathrm{n}=$ número de indivíduos; $\%=$ porcentagem; $T=$ vértebra torácica; $L=$ vértebra lombar; $G=$ graus de fraturas; $K=$ Kappa de Cohen (nível de concordância); IC = intervalo de confiança.

alterações significativas da DMO, resultando em osteoporose secundária, o que corrobora com o nosso estudo, no qual observamos que todos os indivíduos do GDPOC faziam uso de doses acumulativas maiores que $1.000 \mathrm{mg}$ de prednisona ou equivalente. 0 uso de corticoide, tanto inalatório quanto sistêmico, pode causar perda óssea (27), diminuição e perda da força muscular, contribuindo para o surgimento da osteoporose, quando usado de forma prolongada nos tratamentos das exacerbações nesses indivíduos.

Em seu estudo, Creutzberg (28) observou que o uso prolongado de corticoides está diretamente associado à diminuição e à perda de força muscular em indivíduos com DPOC, estimulando a proteólise e inibindo a síntese proteica e o transporte de aminoácidos para as células musculares. Além disso, os 
corticoides e a acidose ativam a via da ubiquitina, conhecida por aumentar a degradação proteica, principalmente nos indivíduos em períodos de exacerbações. A redução da atividade contrátil do músculo influencia o trofismo e o balanço entre síntese e degradação musculares (29).

Kulak et al. (30) observaram que o baixo peso, o sedentarismo, a inatividade física e a fraqueza muscular são fatores de risco muito comuns na DPOC e podem contribuir para o desenvolvimento da osteoporose.

A osteoporose é considerada um problema de saúde pública pela grande prevalência e pelos gastos exorbitantes com o tratamento das fraturas osteoporóticas (31). Classicamente, as fraturas ocorrem no colo do fêmur, vértebras e antebraço (32) sendo que o risco de fraturas é maior após os 50 anos de idade. Em caucasianos, o risco ao longo da vida é de $40 \%$ a 50\% para os indivíduos do sexo feminino e entre $13 \%$ e $22 \%$ para os do sexo masculino (32).

Alguns estudos não têm sido coerentes em apontar um aumento significativo de fraturas nos indivíduos com DPOC $(11,33)$. Enquanto que, Papaioannou et al. (13) encontraram uma prevalência de fraturas vertebrais entre grupos com e sem DPOC, porém os indivíduos com DPOC apresentaram um maior risco de fraturas vertebrais graves. Nossos resultados evidenciaram que 24 indivíduos (71\%) do GDPOC apresentaram fraturas vertebrais osteoporóticas avaliadas por radiografias da coluna toracolombar. VRIES et al. (34) observaram um maior risco de fraturas somente quando os indivíduos apresentaram obstrução grave. Embora os mecanismos envolvidos na gênese da osteoporose e fraturas vertebrais relacionadas à DPOC não sejam claros, certamente eles são complexos e envolvem diversos fatores na sua patogênese, tais como: redução da capacidade de exercício devido à limitação ventilatória, redução da massa muscular esquelética, mudanças na composição corporal, inflamação sistêmica e corticoterapia (35). Quando as fraturas ocorrem, esses indivíduos, que já são debilitados por causas das alterações pulmonares, ficam ainda mais debilitados (35).

Observamos, ainda no presente estudo, que nenhum indivíduo do GC apresentou fraturas vertebrais osteoporóticas, por não estarem expostos aos fatores de risco que pudessem contribuir para o surgimento da osteoporose e das fraturas. Segundo Rochira et al. (8), cerca de 30 a $60 \%$ dos indivíduos do sexo masculino com osteoporose têm uma causa secundária ou associação de fatores de risco que podem ser identificados como fator etiológico. 0 uso de corticosteroides é responsável por $20 \%$ dos casos, o hipogonadismo ocorre em 15 a $20 \%$ dos casos de osteoporose de coluna e o mesmo é cinco vezes mais prevalente em indivíduos do sexo masculino com fratura de fêmur. Já o tabagismo aumenta o risco relativo de fraturas osteoporóticas em 2,3 vezes, independentemente da associação com etilismo (8).

Apesar de na maioria das vezes as fraturas vertebrais osteoporóticas serem assintomáticas, elas oferecem um risco substancial não só para novas fraturas, com consequências clínicas, como também para a saúde geral dos indivíduos com DPOC (36), resultando na incapacidade física, diminuição da estatura, perda de função e piora da qualidade de vida (36).

No entanto, quando presentes, os sintomas das fraturas variam e a dor quando presente é geralmente sentida nas costas, no nível da vértebra fraturada e se espalha em torno daquele nível até a frente do corpo. Muitas vezes a dor é muito intensa, podendo durar dias e até semanas (37). No presente estudo, observou-se que três indivíduos do GDPOC com fraturas vertebrais osteoporóticas relataram dor com intensidade "leve" na região toracolombar.

0 quadro doloroso e a deformidade vertebral podem levar às alterações e dificuldades respiratórias e exacerbações da sintomatologia em indivíduos com DPOC (33), limitando as atividades da vida diária, tais como fazer compras, executar trabalhos domésticos, jardinagem ou permanecer sentado por muito tempo (38). Nos casos mais graves, o tórax é empurrado de tal maneira que as costelas inferiores se encostam na parte de cima dos ossos da pelve, causando um desconforto intenso (38). Além disso, há menos espaço para a expansão dos pulmões, ocasionando dispneia.

Quando a coluna fica muito curva, a pessoa sente dificuldade em manter a cabeça em sua posição normal, o que lhe causa dor cervical e cefaleia. A mudança da posição corporal e suas consequências resultam na perda da autoestima afetando as atividades sociais. Muitos indivíduos temem as quedas, o que restringe ainda mais suas atividades físicas e sociais (38).

Diante do contexto, os indivíduos com tais comorbidades não deverão ser excluídos dos programas fisioterapêuticos de reabilitação pulmonar, dos treinamentos aeróbio, resistido e combinado (39), devendo ser adaptados de acordo com as necessidades 
e capacidades individuais. Os programas de exercício físico influenciam positivamente no equilíbrio, na mobilidade, na coordenação, na resistência muscular e na DMO, reduzindo o número e a gravidade das quedas e da incapacidade física (40).

Cabe destacar como relevância clínica deste estudo a identificação da importância de se utilizar uma avaliação médica e fisioterapêutica cuidadosa e minuciosa da DMO, minimizando o risco de futuras fraturas vertebrais osteoporóticas, piora da capacidade funcional e da qualidade de vida.

\section{Conclusão}

Concluiu-se que os indivíduos com DPOC moderada e grave apresentaram, além do comprometimento pulmonar, diminuição da DMO e fraturas vertebrais osteoporóticas, o que indica a necessidade de se adotar medidas que levem em conta a prevenção da osteoporose nesses pacientes e o diagnóstico precoce desta. Ainda, considerando-se a integralidade das necessidades do indivíduo, estas devem ser avaliadas e atendidas ao se estabelecer programas de intervenção fisioterapêutica, evitando assim, futuras complicações, como agravamento ou surgimento de novas fraturas, e promovendo melhora da capacidade funcional e da qualidade de vida.

\section{Referências}

1. Kanis J, Melton J, Christiansen C, Mackloskey E. The diagnosis of osteoporosis. J Bone Miner Res. 1994; $9(8): 1137-41$.

2. Johnell O, Kanis JA, Oden A, Johansson H, Gluer C, Fujiwara S, et al. A comparison of total hip BMD as a predictor of fracture risk: a meta-analysis. J Bone Mineral Res. 2005;20 Suppl 1:S4.

3. Pacini S, Aterini S, Ruggiero M, Gulisano M. Bone mineral density and anthropometric measures in normal and osteoporotic men. Ital J Anat Embryol. 1999; 104(4):195-200.

4. Halling A, Persson GR, Berglund J, Johansson O, Renvert $\mathrm{S}$. Comparison between the Klemetti index and heel DXA BMD measurements in the diagnosis of reduced skeletal bone mineral density in the elderly. Osteoporos Int. 2005;16(8):999-1003.
5. Stehman-Breen CO, Sherrard D, Walker A, Sadler R, Alem A, Lindberg J. Racial differences in bone mineral density and bone loss among end-stage renal disease patients. Am J Kidney Dis. 1999;33(5):941-6.

6. Oefelein MG, Resnick MI. The impact of osteoporosis in men treated for prostate cancer. Urol Clin North Am. 2004;31(2):313-9.

7. Bano G, Rodin DA, Pazianas M, Nussey SS. Reduced bone mineral density after surgical treatment for obesity. Int J Obes Relat Metab Disord. 1999;23(4):361-5.

8. Rochira V, Balestrieri A, Madeo B, Zirilli L, Granata A, Carani C. Osteoporosis and male age-related hypogonadism: role of sex steroids on bone (patho)physiology. Eur J Endocrinol. 2006;154(2):175-85.

9. Bilezikian JP. Osteoporosis in men. J Clin Endocrinol Metab. 1999;84(10):3431-4.

10. Biskobing DM. COPD and osteoporosis. Chest. 2002; 121(2):609-20.

11. Riancho JA, Macías JG, Arco CD, Amado JA, Freijanes J, Antón MA. Vertebral compression fractures and mineral metabolism in chronic obstructive lung disease. Thorax. 1987;42(12):962-6.

12. McEvoy CE, Ensrud KE, Bender E, Genant HK, Yu W, Griffith JM, Niewoehner DE. Association between corticosteroid use and vertebral fractures in older men with chronic obstructive pulmonary disease. Am J Respir Crit Care Med. 1998;157(3 Pt 1):704-9.

13. Papaioannou A, Parkinson W, Ferko N, Probyn L. Prevalence of vertebral fractures among patients with chronic obstructive pulmonary disease. Osteoporos Int. 2003;14(11):913-7.

14. Soriano JB, Visick GT, Muellerova H, Payvandi N, Hansell AL. Patterns of comorbidities in newly diagnosed COPD and asthma in primary care. Chest. 2005; 128(4):2099-107.

15. Dubois EF, Röder E, Dekhuijzen R, Zwinderman AE, Schweitzer DH. Dual energy x-ray absorptiometry outcomes in male COPD patients after treatment with different glucocorticoid regimens. Chest. 2002; 121(5):1456-63.

16. Rabe KF, Hurd S, Anzueto A, Barnes PJ, Buist SA, Calverley $\mathrm{P}$, et al. Global strategy for the diagnosis, management, and prevention of chronic obstructive pulmonary disease: Gold Executive Summary. Am J Respir Crit Care Med. 2007;176(6):532-55. 
17. Sociedade Brasileira de Pneumologia e Tisiologia. Diretrizes para testes de função pulmonar. J Bras Pneumol. 2002;28 supl. 3:S1-238.

18. National Institute of Health. Consensus development panel on osteoporosis prevention, diagnosis and therapy. Osteoporosis: prevention, diagnosis and therapy. JAMA. 2001;285(6):785-95.

19. European Prospective Osteoporosis Study (EPOS) Group. Incidence of vertebral fracture in Europe: results from the European Prospective Osteoporosis Study (EPOS). J Bone Miner Res. 2002; 17:716-724.

20. Van D, Klift M, De Laet C, Mccloskey EV, Hofman A, Pols HA. The incidence of vertebral fractures in men and women: the Rotterdam study. J Bone Miner Res. 2002;17(4):1051-56.

21. Black DM, Palermo L, Nevitt MC, Cummings S. Defining incident vertebral deformity: a prospective comparison of several approaches. J Bone Miner Res. 1999; 14(1):90-101.

22. Genant HK, Wu CY, Van Kujik C. Vertebral fracture assessment using a quantitative technique. J Bone Miner Res. 1993;8(9):1137-48.

23. Borg G. Escalas de Borg para a dor e o esforço percebido. São Paulo: Manole; 2002.

24. Landis RJ, Koch GG. The measurement of observer agreement for categorical data. Biometrics. 1977; 33(1):159-74

25. Forli L, Halse J, Haug E, Bjortuft O, Vatn M, Kofstad J, et al. Vitamin D deficiency, bone mineral density and weight in patients with advanced pulmonary disease. J Intern Med. 2004;256(1):56-62.

26. Compston JE. Emerging consensus on prevention and treatment of glucocorticoid-induced osteoporosis. Curr Rheumatol Rep. 2007;9(1):78-84.

27. Berry JK, Baum C. Reversal of chronic obstructive pulmonary disease-associated weight loss: are there pharmacological treatment options? Drugs. 2004; 64(10):1041-52.

28. Creutzberg E. Leptin in relation to systemic inflammation and regulation of the energy balance. Am J Respir Crit Care Med. 1999;160(4):1220-6.

29. Jagoe RT, Engelen MPKJ. Muscle wasting and changes in muscle protein metabolism in chronic obstructive pulmonary disease. Eur Respir J Suppl. 2003;46: 52s-63s.
30. Kulak CAM, Bilezikian JP. Osteoporosis: preventive strategies. Int J Fertil Womens Med. 1998;43(2):56-64.

31. Johnell 0, Kanis J. Epidemiology of osteoporotic fractures. Osteoporos Int. 2005;16 Suppl 2:S3-7.

32. Kanis JA. Assessment of fracture risk. Osteoporos Int. 2005;16(6):581-9.

33. Karadag F, Cildag O, Yurekli Y, Gurgey O. Should COPD patients be routinely evaluated for bone mineral density? J Bone Miner Metab. 2003;21(4):242-46.

34. Vries F, Staa TPV, Bracke MSGM, Cooper C, Leufkens HGM, Lammers JWJ. Severity of obstructive airway disease and risk of osteoporotic fracture. Eur Respir J. 2005;25(5);879-84.

35. Goligher JE. Radiologia convencional da osteoporose. In: Bandeira F, Macedo G, Caldas G, Faria M. Osteoporose. Rio de Janeiro: Medsi; 2000. p. 113-26.

36. Ionescu AA, Schoon E. Osteoporosis in chronic obstructive pulmonary disease. Eur Respir J Suppl. 2003; $46: 64 s-75 s$

37. Kanis JA, McClosckey EV. Epidemiology of vertebral osteoporosis. Bone. 1992;13 Suppl 2:S1-10.

38. Lincx Sistemas de Saúde. [acesso 27 set. 2009]. Disponível em: http://www.lincx.com.br/cuidando-de-sua-saude/saude-de-a-a-z/medicina/saude-da-mulher/4745-a-menopausa-o-que-acontece-com-seu-corpo.html.

39. Dourado VZ, Godoy I. Recondicionamento muscular na DPOC: principais intervenções e novas tendências. Rev Bras Med Esporte. 2004;10(4):331-4.

40. Aveiro MC, Navega MT, Granito RN, Rennó ACM, Oishi J. Efeitos de um programa de atividade física no equilíbrio e na força muscular do quadríceps em mulheres osteoporóticas visando uma melhoria na qualidade de vida. Rev Bras Ci Mov. 2004;12(3):33-8.

Recebido: 07/ 12/2010

Received: 12/ 07/2010

Aprovado: 01/08/2011

Approved: 08/01/2011 